\title{
Results of soy-based meal replacement formula on weight, anthropometry, serum lipids \& blood pressure during a 40-week clinical weight loss trial
}

\author{
Kevin R Fontaine ${ }^{1}$, Dongyan Yang2, Gary L Gadbury ${ }^{3}$, Stanley Heshka ${ }^{4}$, \\ Linda G Schwartz ${ }^{4}$, Radha Murugesan ${ }^{4}$, Jennifer L Kraker ${ }^{4}$, Moonseong Heo ${ }^{5}$, \\ Steven B Heymsfield ${ }^{4}$ and David B Allison*2
}

\begin{abstract}
Address: ${ }^{1}$ Division of Rheumatology, Johns Hopkins University School of Medicine, Baltimore, MD, United States, ${ }^{2}$ Department of Biostatistics \& Clinical Nutrition Research Center, University of Alabama at Birmingham, Birmingham, AL, United States, ${ }^{3}$ Department of Mathematics and Statistics, University of Missouri - Rolla, United States, ${ }^{4}$ Obesity Research Center, St. Luke's/Roosevelt Hospital, Institute of Human Nutrition, Columbia University College of Physicians \& Surgeons, New York, New York, United States and ${ }^{5}$ Weill Medical College of Cornell, White Plains, New York, United States

Email: Kevin R Fontaine - kfontai1@jhmi.edu; Dongyan Yang - Dongyan.Yang@ccc.uab.edu; Gary L Gadbury - gadburyg@umr.edu; Stanley Heshka - sh311@columbia.edu; Linda G Schwartz - dg108@columbia.edu; Radha Murugesan -dg108@columbia.edu; Jennifer L Kraker - dg108@columbia.edu; Moonseong Heo - moh2002@med.cornell.edu; Steven B Heymsfield - sbh2@columbia.edu; David B Allison* - DAllison@uab.edu

* Corresponding author
\end{abstract}

This article is available from: http://www.nutritionj.com/content/2/l//4

(C) 2003 Fontaine et al; licensee BioMed Central Ltd. This is an Open Access article: verbatim copying and redistribution of this article are permitted in all media for any purpose, provided this notice is preserved along with the article's original URL.

\begin{abstract}
Background: To evaluate the intermediate-term health outcomes associated with a soy-based meal replacement, and to compare the weight loss efficacy of two distinct patterns of caloric restriction.

Methods: Ninety overweight/obese $\left(28<\mathrm{BMI} \leq 4 \mathrm{l} \mathrm{kg} / \mathrm{m}^{2}\right)$ adults received a single session of dietary counseling and were randomized to either 12 weeks at $1200 \mathrm{kcal} /$ day, 16 weeks at 1500 $\mathrm{kcal} / \mathrm{d}$ and 12 weeks at $1800 \mathrm{kcal} / \mathrm{d}$ (i.e., the $12 / 15 / 18$ diet group), or 28 weeks at $1500 \mathrm{kcal} / \mathrm{d}$ and 12 weeks at $1800 \mathrm{kcal} / \mathrm{d}$ (i.e., the I5/I8 diet group). Weight, body fat, waist circumference, blood pressure and serum lipid concentrations were measured at 4-week intervals throughout the 40week trial.

Results: Subjects in both treatments showed statistically significant improvements in outcomes. A regression model for weight change suggests that subjects with larger baseline weights tended to lose more weight and subjects in the $12 / 15 / 18$ group tended to experience, on average, an additional $0.9 \mathrm{~kg}$ of weight loss compared with subjects in the 15/18 group.
\end{abstract}

Conclusion: Both treatments using the soy-based meal replacement program were associated with significant and comparable weight loss and improvements on selected health variables.

\section{Background}

Obesity is a medically serious [1] and increasingly prevalent condition in the United States [2] associated with increased morbidity [3], mortality rate [4], impaired health related quality of life [5] and reduced life expectancy [6]. Although it is uncertain whether intentional 
weight loss increases longevity [7], it is well documented that losing weight improves health [8] and quality of life [9]. Thus, promoting sustained weight loss remains an important treatment goal for obese individuals. We [10] recently demonstrated that a program involving a soybased meal replacement formula is effective in lowering weight, fat mass and in reducing LDL cholesterol. Because this study was only a 12-week trial, we could not evaluate the intermediate-term effects of the treatment. The primary purpose of this study reported herein was to conduct a trial to evaluate the intermediate-term changes among subjects on weight and several weight-related variables such as blood pressure and serum lipid concentrations. A second purpose of the study was to compare the weight loss efficacy of two distinct recommended patterns of caloric restriction. Finally, because a proportion of our participants were members of the control group in our first study [10], we were able to evaluate whether their prior participation as controls influenced their response to the weight loss intervention.

\section{Methods}

\section{Study Design}

The study is a controlled randomized parallel group 40week trial to compare the intermediate-term weight-loss efficacy of two patterns of prescribed calorie restriction using a soy-based meal replacement product. Subjects were randomly assigned to a sequence of either 12 weeks at $1200 \mathrm{kcal} / \mathrm{day}, 16$ weeks at $1500 \mathrm{kcal} / \mathrm{d}$ and 12 weeks at $1800 \mathrm{kcal} / \mathrm{d}$ (the 12/15/18 diet group), or 28 weeks at $1500 \mathrm{kcal} / \mathrm{d}$ and 12 weeks at $1800 \mathrm{kcal} / \mathrm{d}$ (the 15/18 diet group). Secondary outcomes were body fat, waist circumference, serum lipid concentrations and blood pressure.

\section{Subjects}

Overweight/obese persons with body mass index (BMI; $\mathrm{kg} / \mathrm{m}^{2}$ ) between 28 and 41 (men: $\mathrm{n}=13$ and women: $\mathrm{n}=$ 86) between the ages of 35 and 65 were enrolled in this trial. Exclusion criteria were: weight loss $>5 \mathrm{~kg}$ in the past 3 months, use of weight loss medication within the past 6 weeks, scores above the $90^{\text {th }}$ percentile on the Brief Symptom Inventory (BSI, a screening measure of general psychological functioning), presence of disease not believed to be at least partially the result of obesity and treatable by weight reduction, medical or psychological contraindications as determined by study investigators, or known hypersensitivity to any of the ingredients of the formula, including but not limited to, soy protein. The study was approved by the IRB at St. Luke's/Roosevelt Hospital, and all subjects provided written informed consent. Prior to participation and acceptance into the program, subjects were deemed medically fit for safe weight loss through a physical examination. After meeting eligibility criteria, candidates were randomized via computer-generated pseudo random numbers at a 1:1 allocation ratio, without regard to race or sex, to one of two treatment groups described below. Thirty-seven subjects enrolled in this trial had formerly been the control group for a 12-week weight loss study which has been described elsewhere [10]. An additional 62 were newly recruited from clinic records and advertisements in the local press.

Ninety-nine subjects were randomized, 50 into the 12/15/ 18 treatment group and 49 into the 15/18 treatment group. Sixty-one subjects remained after 12 weeks, 42 after 24 weeks, and 30 subjects completed the 40-week trial.

\section{Treatment Conditions}

All subjects received the Scan Diet meal-replacement formula, instructions for its use, a single session of dietary counseling and a pamphlet describing healthy weight loss practices. The 12/15/18 treatment group was instructed to begin with a $1200 \mathrm{kcal}$ daily diet that consisted of 5 Scan Diet Shakes, 4 exchanges of fruit, 4 exchanges of vegetables and 1 fat exchange. They were given a copy of the Nutricia Scan Diet Meal Plan booklet, describing the diet, as well as a copy of The American Dietetic Association's booklet "Exchange Lists for Weight Management." After 12 weeks the diet instruction was changed to $1500 \mathrm{kcal}$ daily consisting of 3 Scan Diet Shakes, 5 carbohydrate exchanges, 7 exchanges of fruit and vegetables, 5 meat exchanges and 2 fat exchanges. After 16 weeks the diet was increased to an 1,800 kcal/d made up of 2 Scan Diet Shakes, 2 milk, 9 carbohydrate, 8 fruit and vegetable, 8 meat and 3 fat exchanges. The 15/18 group began their weight loss program at the $1500 \mathrm{kcal} /$ day level and was instructed to follow it for 28 weeks. After 28 weeks the diet was increased to the $1800 \mathrm{kcal} / \mathrm{d}$ level, as described above, for the final 12 weeks.

\section{Measures}

Throughout the study, subjects made visits to the clinic at 4 week intervals. At each visit, subjects were checked for compliance and supplied with sufficient meal replacement formula to last until the next scheduled visit, plus one additional week. At each visit anthropometric measures, blood pressure, and psychological wellness assessments were obtained. Blood samples for laboratory assessment included standard blood work at initial screening (i.e., complete blood count and serum lipids) and lipids and serum at weeks 4, 8, 12, 16, 24, 32 and 40. Samples were analyzed by a commercial lab (Quest Diagnostics, Teterboro, NJ). Body weight was measured within $0.1 \mathrm{~kg}$ using a standardized calibrated scale. Height was measured within $.10 \mathrm{~cm}$ using a wall-mounted stadiometer. Body fat was measured through the use of a TANITA bio-impedance analyzer (TBF 305) and waist circumference was taken with a non-distensible tape measure according to published guidelines $[11,12]$. Blood pressure 
was measured after at least 5 minutes rest using a standard mercury sphygmomanometer and appropriately sized cuffs according to the guidelines of the American Heart Association. Side effects and adverse events were assessed by a standardized interview/questionnaire, the Monitoring of Side Effects Scale [13]. The 70-items are answered on a 5 -point scale ranging from $0=$ not present to $4=$ severe.

\section{Statistical Analysis}

The principal aim of the analysis was to compare the effects of the two diet prescriptions over time on the primary and secondary outcomes. We also investigated possible differences in treatment response by the subjects who had been the control group in a previous study with the response of newly recruited subjects.

In initial exploratory analyses, we used two-sample independent t-tests to compare changes from baseline between the two treatment groups at each follow-up visit for, first, only newly recruited subjects and, second, for all subjects. This analysis was used to determine if differences between groups were more apparent for newly recruited subjects. T-tests were also used to assess changes from baseline at each follow-up visit. In this latter analysis, the two treatment groups were combined but separate analyses were run for subjects obtained from our short-term trial [[10], referred to as Study 1], newly recruited subjects, and all subjects together.

We also employed mixed effects regression models with subject as a random effect. This technique accommodated the evaluation of multiple terms in a single model, the evaluation of potentially significant interaction terms, and missing data due to drop-out [14]. These mixed effects models were computed for changes in weight, body fat, waist circumference, total cholesterol (TC), low density lipoprotein cholesterol (LDL), high density lipoprotein cholesterol (HDL), the proportion of TC as HDL (i.e., HDL/TC), triglycerides, and systolic and diastolic blood pressure.

A typical model was of the form,

$Y_{i j}=\beta_{0}+\beta_{1}\left(\right.$ Time $\left._{i j}\right)+\beta_{2}\left(\text { Time }_{i j}\right)^{2}+\beta_{3}\left(\right.$ Base $\left._{i}\right)+\beta_{4}\left(G R P_{i}\right)+$ $\beta_{5}\left(I N 1_{i}\right)+S_{i}+\varepsilon_{i j}$

where the $j$ subscript denotes a follow-up visit, the $i$ subscript denotes subject, and $Y_{i j}$, the dependent variable is the change from baseline (e.g., follow-up weight - baseline weight), Time is the number of weeks since the initial baseline visit, Base is the baseline value of the outcome variable, $G R P=1$ for subjects in the $15 / 18$ group and zero for the $12 / 15 / 18$ group, IN1 = 1 for subjects who participated in the initial study and 0 for newly recruited sub- jects, $S$ is a random effect term for each subject assumed to have a zero mean and variance $\sigma_{S}^{2}, \varepsilon$ represents random errors assumed to be normally distributed with mean zero, variance $\sigma^{2}$, and is assumed to be independent of $S$. Time was coded as a numeric quantity which allowed the development of a functional relationship between Time and change from baseline. Once a model is selected and fitted to the data for a particular outcome variable, the interrelationships between GRP, Time, Base, and IN1 were assessed.

\section{Results}

Baseline characteristics of subjects enrolled in the trial are shown in Table 1. Thirty-six percent of subjects were European-American, 34\% African-American, 20\% HispanicAmerican, and $10 \%$ other or unknown. Adherence to dietary prescription was measured by counting meal replacement packages consumed as a proportion of the number of packages that should have been consumed had the subject used exactly the prescribed amount of soy product, and was expressed as a percentage. Mean group adherence did not differ statistically between the groups and ranged between 48 and $70 \%$ in both groups, except for the final month of the study when it was lower (43-48\%). Regression analysis showed that in both groups amount of weight loss during the trial was positively related to degree of adherence ( $\mathrm{p}=0.0013)$.

The results for weight change are presented in detail below. Following these, results for the remaining variables are presented in summary. Details of all analyses and results for all study variables, as well as figures, can be obtained upon request.

\section{Body Weight}

Figure 1 shows the average change in weight from baseline at each visit for all subjects who made the visit. For the first 4-8 weeks the declines in weight appear to be similar at both levels of caloric restriction but between weeks 12 and 20 the difference between the 1200 and 1500 calorie allocation for the first 12 weeks seems to be reflected in a greater weight loss for the more aggressively restricted group. T-tests showed that the difference only reaches statistical significance among the newly recruited subjects at the 8 week follow-up visit $(P=0.047)$. This greater weight loss is retained until the end of the study in the subjects from Study 1 but not in those newly recruited. Newly recruited subjects in the $12 / 15 / 18$ group achieved their largest mean weight loss at the 16 week visit $(6.44 \mathrm{~kg})$ while those in the $15 / 18$ group had the largest mean weight loss at the 28 week visit $(6.57 \mathrm{~kg})$.

The best fitting mixed effects model for weight change is, 
Table I: Measured Baseline Subject Characteristics (Mean and SD = standard deviation)*

\begin{tabular}{|c|c|c|c|c|c|c|}
\hline \multirow[t]{2}{*}{ Characteristic } & \multicolumn{3}{|c|}{ 12/15/18 Group } & \multicolumn{3}{|c|}{ 15/18 Group } \\
\hline & $\mathbf{N}$ & Mean & (SD) & $\mathbf{N}$ & Mean & (SD) \\
\hline Age, yrs & 50 & 47.7 & $(8.3)$ & 49 & 48.7 & $(8.8)$ \\
\hline Weight, kg & 50 & 91.0 & $(12.5)$ & 49 & 93.5 & $(14.3)$ \\
\hline Body mass index (BMI: $\mathrm{kg} / \mathrm{m}^{2}$ ) & 50 & 34.0 & $(3.5)$ & 49 & 34.6 & $(3.6)$ \\
\hline Total body fat mass $\%$ & 49 & 44.4 & $(5.8)$ & 49 & 44.4 & $(5.2)$ \\
\hline Fat mass, $\mathrm{Kg}$ & 49 & 40.3 & $(8.6)$ & 49 & 41.9 & $(9.7)$ \\
\hline Waist circumference & 49 & 98.0 & $(9.3)$ & 49 & 100.2 & $(9.4)$ \\
\hline Total cholesterol, mg/dL & 48 & 194.8 & $(33.0)$ & 49 & 209.8 & (38.7) \\
\hline LDL cholesterol, mg/dL & 48 & 121.5 & $(28.4)$ & 48 & 134.0 & (37.3) \\
\hline HDL cholesterol, mg/dL & 48 & 54.2 & $(12.6)$ & 49 & $5 \mathrm{I} .4$ & $(11.6)$ \\
\hline Diastolic blood pressure, $\mathrm{mm} \mathrm{Hg}$ & 49 & 80.1 & $(8.9)$ & 49 & 78.2 & $(7.0)$ \\
\hline Systolic blood pressure, $\mathrm{mm} \mathrm{Hg}$ & 49 & 121.9 & $(16.0)$ & 49 & 118.9 & $(14.2)$ \\
\hline Triglyceride, $\mathrm{mg} / \mathrm{dL}$ & 48 & 95.7 & $(57.5)$ & 49 & $|3| . \mid$ & $(83.9)$ \\
\hline
\end{tabular}

* Ns differ because some measures were missing and because the 15/18 group had 49 subjects owing to a clerical error.

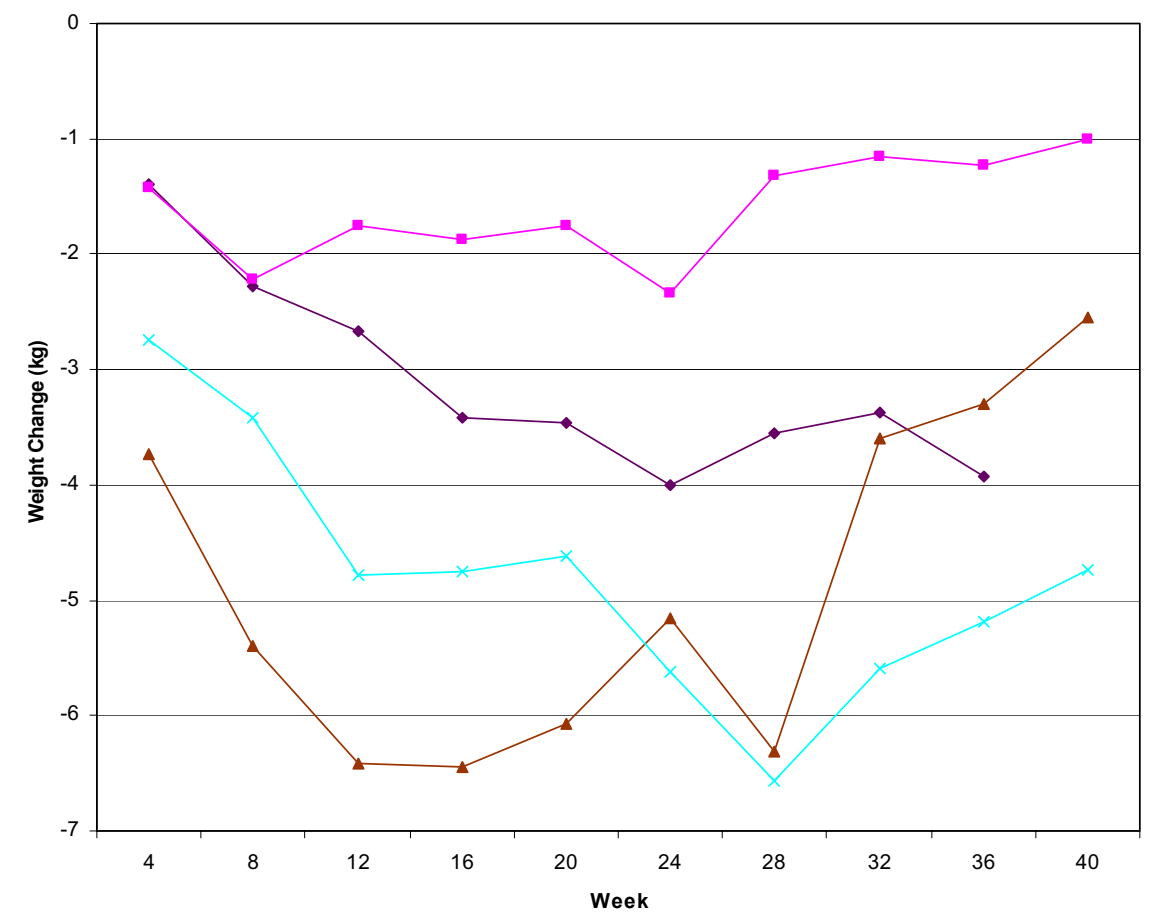

$\multimap 12 / 15 / 18$ Group (subjects from study 1 )

$\rightarrow 15 / 18$ Group (subjects from study 1)

- $12 / 15 / 18$ Group (subjects not from study 1 )

$\times 15 / 18$ Group (subjects not from study 1 )

\section{Figure I}

Changes in weight for subjects from Study I and newly enrolled for the I2/I5/I8 and I5/I8 groups**The left $Y$ axis is the scale of weight change for subjects in study 2 only, while the right $Y$ axis is the scale of weight change for subjects who were in study I. Last time point of I2/I5/I8 group with subjects were in study I is not showing because there were only 4 cases for the point, and the mean was $-7.6 \mathrm{~kg}$, SE was $4.1 \mathrm{~kg}$. 
$\Delta W T=2.12-0.209$ Time $+0.005(\text { Time })^{2}-0.058$ Base + $0.926 G R P+2.117 I N 1+S$

where $\Delta W T$ is the predicted weight change from the fitted model and $S$ is a random effect that is estimated for each subject. The independent predictor variables, Time, (Time) $)^{2}$, Base and IN1 were significant $(\alpha=0.05)$, but GRP was not $(P=0.171)$. No higher order interactions were found to be significant. Time ranged from a minimum of 2.86 weeks (first follow-up visit) to a maximum of 49 weeks (the final follow-up visit).

We interpret the model as follows. Subjects tend to lose weight over time (-0.209 Time) but the loss is less pronounced at later visits. Subjects with larger baseline weights tended to lose more weight. Newly recruited subjects lost, on average, $2.1 \mathrm{~kg}$ more than subjects from Study 1, not surprising since subjects who had been in study 1 entered study 2 already 2.9 kg below their study 1 baseline weight having lost some weight as the control group in the previous study [10].

\section{Body Fat and Waist Circumference}

The mixed effects models for total body fat change indicated that, as for body weight, subjects tend to lose total body fat over time but the loss is less pronounced at later visits due to a positive quadratic effect of time. The rate of change in body fat over time depended on whether subjects had been in the previous study. There were no significant differences between the two treatments on loss of either body fat or total body fat expressed as a percentage at any single follow-up visit.

The best mixed effects model for waist circumference change indicated that subjects' waist circumference decreased over time but the decrease was less pronounced at later visits. Subjects with larger initial waist circumference showed larger decreases and subjects in the 12/15/18 group experienced, on average, an additional $1.729 \mathrm{~cm}$ of waist circumference decrease versus subjects in the 15/18 group. Significant differences were observed between the two treatments at week $4,8,12$, and 16 . In each case, reductions were greater in the $12 / 15 / 18$ group.

\section{Serum lipid}

The mixed effects model for cholesterol indicates that there is an initial overall decrease in cholesterol of a magnitude related to baseline value and that, over time, the cholesterol tends to increase but at a decreasing rate. Subjects in the 12/15/18 group tended to experience, on average, an additional $7.64 \mathrm{mg} / \mathrm{dl}$ reduction in total cholesterol compared with subjects in the $15 / 18$ group.

The models for HDL and LDL cholesterol change, as for total cholesterol, showed an initial decrease of a magni- tude related to baseline value followed by a regain over time, but at a decreasing rate. For LDL, newly recruited subjects decreased $6.764 \mathrm{mg} / \mathrm{dl}$ more than subjects from Study 1. A significant interaction indicates that the rate of LDL change over time depends on the baseline value.

The mixed effects model for HDL/ Total Cholesterol change had only one significant term, the baseline value, with a coefficient indicating that subjects with higher baseline values showed larger decreases in HDL/TC. Ttests on changes from baseline show that, at all time points throughout the study, the HDL/TC improvement from baseline was highly significant $(\mathrm{p}<0.002)$.

Triglyceride changes were not significantly related to any of the individual variables in the mixed effects model. Significant interaction effects indicate that the relation of the baseline value to the amount of triglyceride change depended on the group and whether subjects had served as controls in the prior study.

\section{Blood Pressure}

The mixed effects models for blood pressure change indicate that subjects' blood pressure decreased over time but the decrease is less pronounced at later visits. Subjects with higher baseline values showed larger decreases. Newly recruited subjects tended to decrease more than subjects who had been in Study 1, and subjects in the 12/ $15 / 18$ group tended to experience a greater blood pressure reduction than subjects in the 15/18 group. By t-test we observed significant differences between the groups on diastolic blood pressure at weeks 16 and 28 with the 12/ $15 / 18$ group producing the greater reduction.

\section{Differences from baseline}

On the whole, we observed significant differences on the primary and secondary outcomes when we compared changes at each follow-up clinic visit with baseline values. This pattern is reflected in the significance of the time variable coefficient in most mixed model analyses. Exceptions were observed on triglycerides and SBP, where the pattern of findings was less consistent. The change from baseline was particularly evident in newly recruited subjects; however, in general, it was also apparent when the analysis was carried out on only Study 1 subjects. The smaller effect seen in subjects who had previously served as controls may be a consequence of the fact that they had already lost an average of $2.9 \mathrm{~kg}$ during the previous 3 months.

\section{Discussion}

This 40-week weight loss trial comparing two distinct patterns of caloric restriction indicated that, on the whole, both protocols were associated with fairly similar magnitudes of beneficial weight loss, as well as similar beneficial 
changes on health variables. When newly recruited subjects and previous control subjects were pooled there were no significant differences between the calorie restriction groups on weight loss, body fat, total cholesterol, HDL cholesterol and triglycerides at each assessment. Significant differences were observed on WC, LDL cholesterol and blood pressure at various assessment points, and, in these cases, the magnitude of the change was greater for the $12 / 15 / 18$ group.

The exact implications of the observed cholesterol changes are not immediately apparent because of the simultaneous variation in the total and fractions of cholesterol. Decreases seen in total and LDL levels, which would be interpreted as beneficial, are accompanied by a decrease in HDL, which is known to have a protective effect. One resolution is to use a combination measure such as the HDL/total cholesterol index which has been shown to have stronger associations with coronary heart disease than the individual cholesterol components [15]. In this study the HDL/total cholesterol index was significantly improved over baseline in both groups at every time point.

The results of the mixed models were generally consistent: no matter the health variable in question, subjects tend to have a desirable change in the outcome over time, with the largest changes occurring earlier in the trial. Moreover, subjects with less desirable baseline values (e.g., larger waist circumference) tended to show a change of greater magnitude. Finally, subjects in the $12 / 15 / 18$ group tended to experience, on some measures, additional change on the outcome versus subjects in the 15/18 group.

There was also a tendency for newly recruited subjects to show greater improvements than subjects who had previously served as controls. The IN1 variable was positive in all models although it only occasionally reached conventional significance levels. This may be the result of the weight loss that had previously occurred in the control group, making it more difficult for subjects to lose additional weight in this follow-up study. Study designs which call for re-randomization of subjects who previously served as controls should consider the possibility of diminished effects.

This study has limitations. First, this 40 -week trial did not include a non treatment control condition. Thus, we were unable to evaluate the effects of the two treatments compared with the absence of treatment. In this regard, however, our previous study (10) demonstrated that, over 12weeks, assignment to the soy-based meal replacement program caused reductions in weight and improvements on health variables compared with receiving only dietary advice. In addition, the outcome measures are substantially improved during and at the end of the study period compared to baseline values. Nonetheless, the absence of a no treatment control group in this trial limits the conclusions that can be drawn.

Second, because of the duration of the study and the minimal intervention offered, only $30 \%$ of the subjects completed the entire trial. Although high attrition rates are not uncommon even in shorter-term weight loss trials [16], it raises the possibility that our results may not be representative of all persons who take part in this, or a similar, weight loss protocol.

\section{Conclusions}

This 40 -week trial indicates that a reduced calorie deficit diet that includes a soy-based meal replacement shake produces significant changes on body weight and on selected health variables. Adherence to the prescribed degree of soy product usage remained relatively constant throughout, except for the final month. Greater adherence was associated with greater weight loss during the trial. Finally, both patterns of caloric restriction were effective in promoting weight loss and were associated with comparable levels of weight loss over the course of the trial. In general, this was also the case with regard to important health variables such as blood pressure and serum lipid concentrations.

\section{Competing interests}

This study was funded by NutriPharma, and Dr. Allison is a consultant to the United Soybean Board.

\section{Authors' Contributions}

KF assisted writing the manuscript and provided oversight in producing the final document. DY participated in data management, data analysis, and interpretation. GG participated in data analysis and interpretation. LS, RM, and JK delivered the intervention. SH provided oversight of study execution and drafted parts of the manuscript. MH participated in data management and data analysis. SBH assisted in interpretation of study data. DB designed the trial, oversaw study execution, data analysis, and interpretation. All authors read and approved the final manuscript.

\section{References}

I. Allison DB, Pi-Sunyer FX: Obesity Treatment: Examining the Premises. Endo Prac 1995, I:353-364.

2. Flegal KM, Carroll MD, Ogden CL, Johnson CL: Prevalence and trends in obesity among US adults, 1999-2000. JAMA 2002, 288: $1728-1732$.

3. Pi-Sunyer FX: Comorbidities of overweight and obesity: current and research issues. Med Sci Sports Med 1999, Suppl 3 I:S602-S608.

4. Allison DB, Fontaine KR, Manson JE, Stevens J, Vanltallie TB: How many deaths are attributable to obesity? JAMA I999, 282:1530-1538. 
5. Heo M, Allison DB, Faith MS, Zhu S, Fontaine KR: Obesity and health-related quality of life: the mediating effects of joint pain and comorbidities. Obes Res 2003, I I:209-2I6.

6. Fontaine KR, Redden D, Wang C, Westfall A, Allison DB: Years of life lost due to obesity. JAMA 2003, 289:187-193.

7. Yang D, Fontaine KR, Wang C, Allison DB: Con: Weight loss causes increased mortality. Obes Revs 2003, 4:9-16.

8. Fontaine $K R$, Allison $D B$ : Does intentional weight loss affect mortality rate? Eating Behav 200I, 2:87-95.

9. Fontaine KR, Barofsky I: Obesity and health-related quality of life. Obes Revs 200I, 2:173-182.

10. Allison DB, Gadbury G, Schwartz LG, Murugesan R, Kraker JL Heshka S, Fontaine KR, Heymsfield SB: A novel soy-based meal replacement formula for weight loss among obese individuals: a randomized controlled clinical trial. Euro J Clin Nutri 2003, 57:5। 4-522.

II. Heymsfield SB, Wang Z, Visser M, Gallagher D, Pierson RN Jr: Techniques used in the measurement of body composition: an overview with emphasis on bioelectrical impedance analysis. Am J Clin Nutri 1996, 64(3 Suppl):478S-484S.

12. Kalachnik JE: Medication monitoring procedures: Thou shall, here's how. In: Pharmacotherapy and Mental Retardation Edited by: Gadow KD, Poling AG. Boston: College-Hill Press; 1985:244-245.

13. Lohman TG, Roche AF, Martorell R: Anthropometric standardization reference manual Champaign, IL: Human Kinetics Books; 1988.

14. Gadbury GL, Coffey CS, Allison DB: Modern statistical methods for handling missing repeated measurements in obesity trial data: Beyond LOCF. Obes Revs 2003, 4: I75-184.

15. Kannel WB: The Framingham Study: Its 50-year legacy and future promise. J Atheroscler Thromb 2000, 6:60-66.

16. Honas J], Early JL, Frederickson DD, O'Brien MS: Predictors of attrition in a large clinic-based weight-loss program. Obes Res 2003, I I:888-894.

Publish with Bio Med Central and every scientist can read your work free of charge

"BioMed Central will be the most significant development for disseminating the results of biomedical research in our lifetime. "

Sir Paul Nurse, Cancer Research UK

Your research papers will be:

- available free of charge to the entire biomedical community

- peer reviewed and published immediately upon acceptance

- cited in PubMed and archived on PubMed Central

- yours - you keep the copyright
BioMedcentral 